\title{
Impaired fasting glucose: a risk factor for atrial fibrillation and heart failure
}

\author{
Viktor Lind ${ }^{1,2^{*}} \mathbb{D}$, Niklas Hammar ${ }^{3}$, Pia Lundman ${ }^{1,2}$, Leif Friberg ${ }^{1}$, Mats Talbäck ${ }^{3}$, Göran Walldius ${ }^{3}$ and \\ Anna Norhammar ${ }^{4,5}$
}

\begin{abstract}
Background: Dysglycaemia is associated with overall cardiovascular disease even at prediabetes levels. The aim of this study was to explore the association between glucose levels and future risk of developing atrial fibrillation and heart failure, respectively.

Methods: In this prospective cohort study subjects from the Swedish AMORIS-cohort with fasting glucose from health examinations 1985-1996 without previous cardiovascular disease $(N=294,057)$ were followed to 31 December 2011 for incident atrial fibrillation or heart failure. Cox proportional hazard models with attained age as timescale and adjustments for sex, cholesterol, triglycerides, and socioeconomic status were used to estimate hazard ratios by glucose categorized groups (normal glucose 3.9-6.0 mmol/L, impaired fasting glucose; 6.1-6.9 mmol/L, undiagnosed diabetes $\geq 7.0 \mathrm{mmol} / \mathrm{L}$, and diagnosed diabetes).

Results: During a mean follow-up time of 19.1 years 28,233 individuals developed atrial fibrillation and 25,604 developed heart failure. The HR for atrial fibrillation was 1.19 (95\% confidence interval 1.13-1.26) for impaired fasting glucose, 1.23 (1.15-1.32) for undiagnosed diabetes and 1.30 (1.21-1.41) for diagnosed diabetes. Corresponding figures for heart failure were; 1.40 (1.33-1.48), 2.11 (1.99-2.23), 2.22 (2.08-2.36) respectively. In a subset with BMI data (19\%), these associations were attenuated and for atrial fibrillation only remained statistically significant among subjects with diagnosed diabetes (HR 1.25; 1.02-1.53).
\end{abstract}

Conclusions: Fasting glucose at prediabetes levels is associated with development of atrial fibrillation and heart failure. To some extent increased BMI may drive this association.

Keywords: Prediabetes, Impaired fasting glucose, Dysglycaemia, Diabetes, Cardiovascular disease, Atrial fibrillation, Heart failure

\section{Background}

Diabetes mellitus is associated with an increased risk of cardiovascular disease and compromised longevity [1]. This increased risk is present already at prediabetes levels [2]. Globally, diabetes is estimated to affect 463 million adults, with one-third to one half being undiagnosed, and with a projected increase in prevalence to 578 million in

*Correspondence: viktor.lind@ki.se

${ }^{1}$ Division of Cardiovascular Medicine, Department of Clinical Sciences, Danderyd Hospital, Karolinska Institutet, 18288 Stockholm, Sweden

Full list of author information is available at the end of the article the year 2030 [3]. One of the most severe cardiovascular complications associated with diabetes is heart failure [4], with diabetes increasing the risk for heart failure by up to two times when risk factors are well-controlled and substantially higher if uncontrolled $[1,5,6]$. Furthermore, diabetes has been implicated as a risk factor for incident atrial fibrillation, as well as having a negative impact on prognosis of this disease [6-10]. The cardiovascular risk associated with prediabetes is mostly described for cardiovascular mortality, but an association has also been suggested for heart failure [11, 12] and atrial fibrillation [13-16]. original author(s) and the source, provide a link to the Creative Commons licence, and indicate if changes were made. The images or other third party material in this article are included in the article's Creative Commons licence, unless indicated otherwise in a credit line to the material. If material is not included in the article's Creative Commons licence and your intended use is not permitted by statutory regulation or exceeds the permitted use, you will need to obtain permission directly from the copyright holder. To view a copy of this licence, visit http://creativecommons.org/licenses/by/4.0/. The Creative Commons Public Domain Dedication waiver (http://creativeco mmons.org/publicdomain/zero/1.0/) applies to the data made available in this article, unless otherwise stated in a credit line to the data. 
The aim of this study was to explore the association between glucose levels, including those below the current diagnostic threshold of diabetes, and future risk of developing atrial fibrillation and heart failure, respectively, among individuals without previous cardiovascular disease.

\section{Methods}

\section{Study population and data sources}

This prospective cohort study included subjects from the Swedish Apolipoprotein MOrtality RISk (AMORIS) cohort [17]. The cohort was initially set up to study associations between apolipoprotein (apo) B and apoA-1 and cardiovascular disease, but has since then been extensively utilized to study metabolic disturbances and inflammation and the risk of several chronic diseases including type 2 diabetes [18]. It consists of 812073 unselected individuals predominantly from the larger Stockholm area referred for laboratory testing either via routine health check-ups from occupational health care or from primary health care during the time-period 1985 to 1996 . From this cohort we included study subjects 30-84 years of age with a measurement of fasting glucose, triglycerides, and total cholesterol at the same examination (index examination). Subjects with a history of atrial fibrillation, heart failure, ischemic heart disease, stroke or revascularization at the index examination were excluded (See Additional file 1: Table S1). The final study population included 294057 subjects (Fig. 1). Information on co-morbidities and mortality prior to and following the index examination was gained through linkage to the National Patient register going back to 1964 regionally, to 1972 in Stockholm county, and to 1987 nationally, the National Cancer Register going back to 1958 and the National Cause of Death Register going back to 1961. For record linkage we utilized the unique Swedish personal identification number available in all relevant registers.

\section{Laboratory measurements}

Information on biomarkers reflecting metabolic abnormalities and inflammation was obtained from the index examination. All biomarkers were analyzed at the time of the health examination in fresh blood at the CALAB medical laboratory (Stockholm, Sweden). For details on analytic methods, see Additional File 1.

\section{Follow-up}

Subjects were followed for incident atrial fibrillation and incident heart failure respectively using the Patient Register and the Cause of Death Register. ICD-codes were registered by physicians from inpatient care, emergency care and, from 2001, from specialized outpatient care (See Additional File 1: Table S2). Follow-up started at the

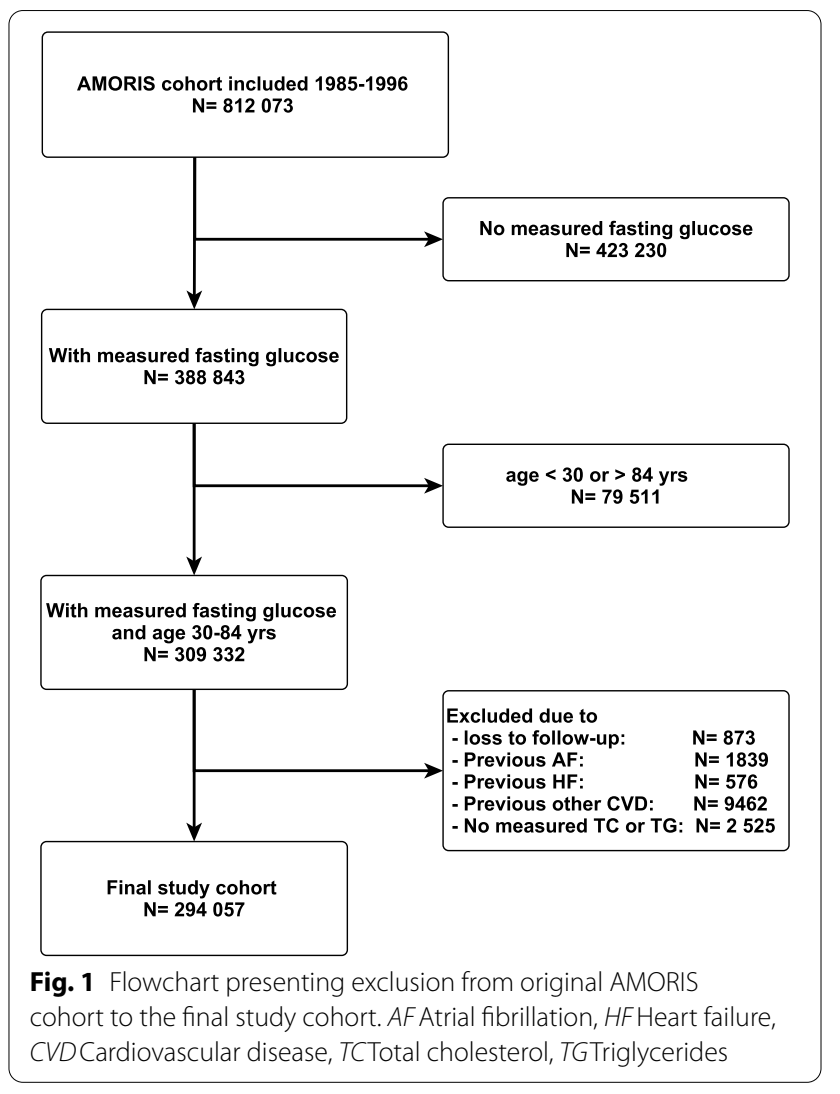

index examination and ended at the first recorded diagnosis of atrial fibrillation or heart failure respectively, death, migration or end of follow-up at December 31, 2011.

\section{Definitions}

Outcomes: First hospitalization or specialized outpatient visit or main cause of death due to atrial fibrillation or heart failure respectively. Combined event was defined as first event of either atrial fibrillation or heart failure.

Exposure: Subjects free of diagnosed diabetes at baseline were categorized into four groups by fasting glucose measurements at the index examination; (i) Low (<3.9 mmol/L); (ii) Normal $(3.9-6.0 \mathrm{mmol} / \mathrm{L})$; (iii) Impaired Fasting Glucose (6.1-6.9 mmol/L in accordance to the definition by the WHO)); [19] (iv) Undiagnosed diabetes $(\geq 7.0 \mathrm{mmol} / \mathrm{L})$.

Diagnosed prevalent diabetes was defined as a registered ICD-code of diabetes prior to the index examination in the National Diabetes Register or the Patient Register (250) or as self-reported diabetes in linked research studies [17].

Prediabetes was defined as a fasting glucose between 6.1 and $6.9 \mathrm{mmol} / \mathrm{L}$, but not on $2 \mathrm{~h}$ oral glucose tolerance test or HbA1c since these were not available. 
eGFR (estimated glomerular filtration rate) was calculated using the Chronic Kidney Disease Epidemiology Collaboration formula (CKD-EPI) [20].

BMI (Body Mass Index) calculated as $\mathrm{kg} / \mathrm{m}^{2}$ was attained from either the index examination, earlier examinations, the National Medical Birth Register, the Diabetes Register or linked research studies. We used BMI within a time window of up to ten years prior to the index examination.

Co-morbidities: Co-morbidities were identified from ICD-codes in the Patient Register or from diagnosed malignant cancer in the Cancer Register (See Additional File 1: Table S3).

Socioeconomic status: Information on socioeconomic status was obtained from the nearest national population and housing census 1970-1990 prior to the index examination. Socioeconomic status was classified as white- or blue-collar worker based on a Swedish Socioeconomic Index (SEI).

\section{Statistical analysis}

Baseline characteristics were described using frequencies and percentages for categorical variables and interquartile range and median values for continuous variables. Incidences were calculated as the numbers of new events divided by person years at risk. Cox proportional hazards regression with attained age as timescale was used to estimate hazard ratios (HR) together with 95\% confidence intervals (CI). Adjustments were made for sex and with additional adjustment for total cholesterol, triglycerides and socioeconomic status. Corresponding sensitivity analysis was performed with additional adjustment for BMI in a sub-population. To illustrate the possible impact of competing events, the incidence proportion of the first event of atrial fibrillation, heart failure and death due to other causes, respectively, were calculated by age groups across a 25-year follow-up period and shown graphically. Proportional hazard ratios for atrial fibrillation and heart failure respectively, in relation to increasing glucose levels were estimated using restricted cubic splines together with $95 \%$ confidence intervals (mean glucose equal $5 \mathrm{mmol} / \mathrm{L}$ served as the reference with $\mathrm{HR}$ of 1.0).

Statistical analyses were conducted using STATA version 14.2 (StataCorp LLC, College Station, TX, USA).

\section{Results}

The cohort had a median age of 47 years, $46 \%$ were women and $92 \%$ were born in a Nordic country. The mean follow-up time was 19.1 years. The overall median fasting glucose was $4.8 \mathrm{mmol} / \mathrm{L}$ (IQR $4.5-5.2 \mathrm{mmol} / \mathrm{L}$ ).
Impaired fasting glucose and diabetes was associated with higher age and male sex (Table 1). Triglyceridelevels were increased among subjects with impaired fasting glucose, diagnosed diabetes and with undiagnosed diabetes compared to those with normal glucose levels. The apo-B/apo-A1 ratio was also higher in all groups with elevated glucose levels. The overall proportion of subjects with an eGFR below $60 \mathrm{ml} / \mathrm{min} / 1.73$ $\mathrm{m}^{2}$ was $3.5 \%$, among those with normal glucose levels $3.2 \%$, and among subjects with undiagnosed diabetes $10.3 \%$. Information on BMI was available in only $19 \%$ of subjects. This subgroup had similar background characteristics as the total cohort (See Additional File 1: Table S4). The highest mean BMI was found in the group with previously undiagnosed diabetes.

\section{Outcome}

During the follow-up period there were 28233 new cases of atrial fibrillation, 25604 new cases of heart failure and 42083 combined events observed. Elevated fasting glucose was associated with an increased risk for atrial fibrillation, heart failure, and a combined event respectively. The incidence of atrial fibrillation was increased among all groups with elevated glucose measurements (Fig. 2). The highest incidence was found in the group with already diagnosed diabetes followed by the groups undiagnosed diabetes and impaired fasting glucose. The incidence of heart failure followed the same pattern although with higher hazard ratio estimates.

Further adjustment for BMI (Table 2) attenuated the associations for both outcomes and in particular for atrial fibrillation, where the association only remained statistically significant among subjects with diagnosed diabetes (HR 1.25; 1.02-1.53).

The spline curves (HR and 95\% CI) for atrial fibrillation and heart failure showed a continuous increased risk of both incident atrial fibrillation and incident heart failure with successively increasing glucose levels (Fig. 3). At a fasting glucose of $6.1 \mathrm{mmol} / \mathrm{L}$ the HR $(95 \%$ CI) for atrial fibrillation was 1.09 (1.07-1.11).

Figure 4 illustrates the incidence proportions of atrial fibrillation, heart failure and death for men and women by age groups and follow-up time. Among those below the age of 65 years at inclusion atrial fibrillation occurred more frequently as first event without previous heart failure, whereas among those aged 65 years and older atrial fibrillation and heart failure occurred as first event in approximately equal proportions. In the oldest age group mortality from other causes was common and at the end of the follow-up most subjects had experienced at least one of these outcomes. 
Table 1 Baseline characteristics

\begin{tabular}{|c|c|c|c|c|c|c|c|}
\hline & $\mathrm{N}$ & $\begin{array}{l}\text { Total } \\
\mathrm{N}=294057\end{array}$ & $\begin{array}{l}\text { Low } \\
<3.9 \mathrm{mmol} / \mathrm{L} \\
\mathrm{n}=7408(2.5 \%)\end{array}$ & $\begin{array}{l}\text { Normal } \\
3.9-6.0 \mathrm{mmol} / \mathrm{L} \\
\mathrm{n}=266943(90.8 \%)\end{array}$ & $\begin{array}{l}\text { IFG } \\
6.1-6.9 \mathrm{mmol} / \mathrm{L} \\
n=9682(3.3 \%)\end{array}$ & $\begin{array}{l}\text { Undiagnosed DM } \\
\geq 7.0 \mathrm{mmol} / \mathrm{L} \\
\mathrm{n}=5457(1.9 \%)\end{array}$ & $\begin{array}{l}\text { Diagnosed DM } \\
n=4567(1.6 \%)\end{array}$ \\
\hline Age (years) & 294057 & $\begin{array}{l}47 \\
(40-56)\end{array}$ & $\begin{array}{l}43 \\
(36-51)\end{array}$ & $\begin{array}{l}47 \\
(39-55)\end{array}$ & $\begin{array}{l}54 \\
(46-61)\end{array}$ & $\begin{array}{l}57 \\
(49-64)\end{array}$ & $\begin{array}{l}53 \\
(45-60)\end{array}$ \\
\hline Sex, female & 294057 & $\begin{array}{l}135562 \\
(46.1)\end{array}$ & $\begin{array}{l}4382 \\
(59.2)\end{array}$ & $124497(46.6)$ & $\begin{array}{l}3300 \\
(34.1)\end{array}$ & $\begin{array}{l}1646 \\
(30.2)\end{array}$ & $\begin{array}{l}1737 \\
(38.0)\end{array}$ \\
\hline Blue collar worker & 286058 & $\begin{array}{l}157549 \\
(55.1)\end{array}$ & $\begin{array}{l}4090 \\
(56.8)\end{array}$ & $\begin{array}{l}142258 \\
(54.7)\end{array}$ & $\begin{array}{l}5464 \\
(58.3)\end{array}$ & $\begin{array}{l}3084 \\
(59.6)\end{array}$ & $\begin{array}{l}2653 \\
(60.1)\end{array}$ \\
\hline $\begin{array}{l}\text { Born in Nordic coun- } \\
\text { tries }\end{array}$ & 294057 & $\begin{array}{l}270242 \\
(92.1)\end{array}$ & $\begin{array}{l}6854 \\
(92.5)\end{array}$ & $\begin{array}{l}245489 \\
(92.0)\end{array}$ & $\begin{array}{l}8878 \\
(91.7)\end{array}$ & $\begin{array}{l}4884 \\
(89.5)\end{array}$ & $\begin{array}{l}4147 \\
(90.8)\end{array}$ \\
\hline $\begin{array}{l}\text { Referred from occupa- } \\
\text { tional health care }\end{array}$ & 259549 & $\begin{array}{l}193573 \\
(74.6)\end{array}$ & $\begin{array}{l}5187 \\
(79.0)\end{array}$ & $\begin{array}{l}177643 \\
(75.4)\end{array}$ & $\begin{array}{l}5585 \\
(65.6)\end{array}$ & $\begin{array}{l}2710 \\
(57.2)\end{array}$ & $\begin{array}{l}2448 \\
(59.9)\end{array}$ \\
\hline BMI (kg/m2) & 55390 & $\begin{array}{l}24.0 \\
(21.8-26.5)\end{array}$ & $\begin{array}{l}22.4 \\
(20.6-24.6)\end{array}$ & $\begin{array}{l}24.0 \\
(21.8-26.3)\end{array}$ & $\begin{array}{l}26.5 \\
(24.2-29.5)\end{array}$ & $\begin{array}{l}28.0 \\
(25.5-30.9)\end{array}$ & $\begin{array}{l}26.6 \\
(23.4-29.8)\end{array}$ \\
\hline Kidney disease & 294057 & $\begin{array}{l}288 \\
(0.1)\end{array}$ & $\begin{array}{l}9 \\
(0.1)\end{array}$ & $\begin{array}{l}257 \\
(0.1)\end{array}$ & $\begin{array}{l}9 \\
(0.1)\end{array}$ & $\begin{array}{l}2 \\
(0.0)\end{array}$ & $\begin{array}{l}11 \\
(0.2)\end{array}$ \\
\hline Liver disease & 294057 & $\begin{array}{l}1102 \\
(0.4)\end{array}$ & $\begin{array}{l}38 \\
(0.5)\end{array}$ & $\begin{array}{l}862 \\
(0.3)\end{array}$ & $\begin{array}{l}65 \\
(0.7)\end{array}$ & $\begin{array}{l}51 \\
(0.9)\end{array}$ & $\begin{array}{l}86 \\
(1.9)\end{array}$ \\
\hline Asthma/COPD & 294057 & $\begin{array}{l}2026 \\
(0.7)\end{array}$ & $\begin{array}{l}63 \\
(0.9)\end{array}$ & $\begin{array}{l}1740 \\
(0.7)\end{array}$ & $\begin{array}{l}93 \\
(1.0)\end{array}$ & $\begin{array}{l}55 \\
(1.0)\end{array}$ & $\begin{array}{l}75 \\
(1.6)\end{array}$ \\
\hline History of cancer & 294057 & $\begin{array}{l}6550 \\
(2.2)\end{array}$ & $\begin{array}{l}131 \\
(1.8)\end{array}$ & $\begin{array}{l}5812 \\
(2.2)\end{array}$ & $\begin{array}{l}287 \\
(3.0)\end{array}$ & $\begin{array}{l}196 \\
(3.6)\end{array}$ & $\begin{array}{l}124 \\
(2.7)\end{array}$ \\
\hline Mitral stenosis & 294057 & $\begin{array}{l}34 \\
(0)\end{array}$ & 0 & $\begin{array}{l}30 \\
(0)\end{array}$ & $\begin{array}{l}1 \\
(0)\end{array}$ & $\begin{array}{l}2 \\
(0)\end{array}$ & $\begin{array}{l}1 \\
(0)\end{array}$ \\
\hline $\begin{array}{l}\text { Mechanical } \\
\text { valve-replacement }\end{array}$ & 294057 & $\begin{array}{l}25 \\
(0)\end{array}$ & 0 & $\begin{array}{l}24 \\
(0)\end{array}$ & $\begin{array}{l}1 \\
(0)\end{array}$ & 0 & 0 \\
\hline $\begin{array}{l}\text { Fasting glucose, } \\
\mathrm{mmol} / \mathrm{L}\end{array}$ & 294057 & $\begin{array}{l}4.8 \\
(4.5-5.2)\end{array}$ & $\begin{array}{l}3.7 \\
(3.5-3.8)\end{array}$ & $\begin{array}{l}4.8 \\
(4.5-5.2)\end{array}$ & $\begin{array}{l}6.3 \\
(6.2-6.6)\end{array}$ & $\begin{array}{l}8.2 \\
(7.3-10.4)\end{array}$ & $\begin{array}{l}8.3 \\
(6.2-11.9)\end{array}$ \\
\hline Fructosamine, mmol/L & 242205 & $\begin{array}{l}2.1 \\
(2.0-2.2)\end{array}$ & $\begin{array}{l}2.0 \\
(1.9-2.2)\end{array}$ & $\begin{array}{l}2.1 \\
(2.0-2.2)\end{array}$ & $\begin{array}{l}2.2 \\
(2.0-2.3)\end{array}$ & $\begin{array}{l}2.5 \\
(2.2-2.9)\end{array}$ & $\begin{array}{l}2.6 \\
(2.3-3.1)\end{array}$ \\
\hline $\begin{array}{l}\text { Total cholesterol, } \\
\mathrm{mmol} / \mathrm{L}\end{array}$ & 294057 & $\begin{array}{l}5.7 \\
(5.0-6.4)\end{array}$ & $\begin{array}{l}5.4 \\
(4.7-6.1)\end{array}$ & $\begin{array}{l}5.7 \\
(5.0-6.4)\end{array}$ & $\begin{array}{l}6.0 \\
(5.3-6.8)\end{array}$ & $\begin{array}{l}6.0 \\
(5.3-6.8)\end{array}$ & $\begin{array}{l}5.8 \\
(5.0-6.6)\end{array}$ \\
\hline $\begin{array}{l}\text { Triglyceride level, } \\
\mathrm{mmol} / \mathrm{L}\end{array}$ & 294057 & $\begin{array}{l}1.0 \\
(0.7-1.5)\end{array}$ & $\begin{array}{l}0.9 \\
(0.6-1.2)\end{array}$ & $\begin{array}{l}1.0 \\
(0.7-1.5)\end{array}$ & $\begin{array}{l}1.5 \\
(1.0-2.2)\end{array}$ & $\begin{array}{l}1.9 \\
(1.3-2.8)\end{array}$ & $\begin{array}{l}1.5 \\
(1.0-2.4)\end{array}$ \\
\hline Apo-B/Apo-A1 ratio & 294057 & $\begin{array}{l}0.89 \\
(0.71-1.10)\end{array}$ & $\begin{array}{l}0.82 \\
(0.67-1.01)\end{array}$ & $\begin{array}{l}0.88 \\
(0.71-1.09)\end{array}$ & $\begin{array}{l}0.96 \\
(0.79-1.21)\end{array}$ & $\begin{array}{l}1.05 \\
(0.85-1.28)\end{array}$ & $\begin{array}{l}0.97 \\
(0.78-1.22)\end{array}$ \\
\hline Haemoglobin, g/L & 73119 & $\begin{array}{l}141.0 \\
(133.0-150.0)\end{array}$ & $\begin{array}{l}138.0 \\
(129.0-147.0)\end{array}$ & $\begin{array}{l}141.0 \\
(132.0-150.0)\end{array}$ & $\begin{array}{l}146.0 \\
(138.0-154.0)\end{array}$ & $\begin{array}{l}149.0 \\
(140.0- \\
157.0)\end{array}$ & $\begin{array}{l}145.0 \\
(136.0-154.0)\end{array}$ \\
\hline WBC, $10 \wedge 9 / \mathrm{L}$ & 67806 & $\begin{array}{l}6.1 \\
(5.1-7.4)\end{array}$ & $\begin{array}{l}6.1 \\
(5.1-7.6)\end{array}$ & $\begin{array}{l}6.1 \\
(5.1-7.3)\end{array}$ & $\begin{array}{l}6.6 \\
(5.6-7.9)\end{array}$ & $\begin{array}{l}6.9 \\
(5.8-8.3)\end{array}$ & $\begin{array}{l}6.6 \\
(5.5-8.0)\end{array}$ \\
\hline $\begin{array}{l}\text { Subjects with eGFR } \\
\text { below } 60 \mathrm{ml} / \mathrm{min} / 1.73 \\
\mathrm{~m}^{2}\end{array}$ & 283448 & $10173(3.5)$ & $180(2.4)$ & $8435(3.2)$ & $698(7.2)$ & $559(10.3)$ & $301(6.6)$ \\
\hline Uric acid, umol/L & 278499 & $\begin{array}{l}287.0 \\
(240.0-337.0)\end{array}$ & $\begin{array}{l}262.0 \\
(218.0-311.0)\end{array}$ & $\begin{array}{l}286.0 \\
(239.0-335.0)\end{array}$ & $\begin{array}{l}335.0 \\
(285.0-389.0)\end{array}$ & $\begin{array}{l}321.0 \\
(271.0-380.0)\end{array}$ & $\begin{array}{l}286.0 \\
(235.0-345.0)\end{array}$ \\
\hline $\mathrm{CRP}, \mathrm{mg} / \mathrm{L}$ & 139257 & $\begin{array}{l}4.0 \\
(1.0-6.0)\end{array}$ & $\begin{array}{l}3.0 \\
(1.0-6.0)\end{array}$ & $\begin{array}{l}4.0 \\
(1.0-6.0)\end{array}$ & $\begin{array}{l}4.0 \\
(3.0-7.0)\end{array}$ & $\begin{array}{l}4.0 \\
(2.0-8.0)\end{array}$ & $\begin{array}{l}4.0 \\
(2.0-7.0)\end{array}$ \\
\hline Haptoglobin, g/L & 234448 & $\begin{array}{l}1.0 \\
(0.9-1.2)\end{array}$ & $\begin{array}{l}1.0 \\
(0.8-1.2)\end{array}$ & $\begin{array}{l}1.0 \\
(0.9-1.2)\end{array}$ & $\begin{array}{l}1.1 \\
(1.0-1.4)\end{array}$ & $\begin{array}{l}1.2 \\
(1.0-1.4)\end{array}$ & $\begin{array}{l}1.1 \\
(0.9-1.3)\end{array}$ \\
\hline
\end{tabular}

Categorical variables presented as absolute and relative (percentages) frequencies and continuous variables as median and interquartile range IFG Impaired fasting glucose, DMDiabetes mellitus, COPDChronic obstructive pulmonary disease, WBCWhite blood cells

\section{Discussion}

In this long-term follow-up of atrial fibrillation and heart failure in subjects free from cardiovascular disease at baseline there are three major findings. First, that increasing glucose values are associated with an increased risk of future development of both atrial fibrillation and 


\begin{tabular}{|c|c|c|c|c|c|}
\hline \multirow{10}{*}{ 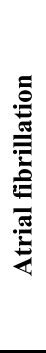 } & Glucose level & PYR & Events & Adjustment & HR $(95 \% \mathrm{CI})$ \\
\hline & \multirow{2}{*}{ Low } & \multirow{2}{*}{147692} & \multirow{2}{*}{490} & Age adj. & $0.98(0.90-1.07)$ \\
\hline & & & & Multi adj. & $0.98(0 \cdot 90-1 \cdot 07)$ \\
\hline & Normal & 5122588 & 24689 & Ref. & $1 \cdot 00$ \\
\hline & \multirow{2}{*}{ IFG } & \multirow{2}{*}{161570} & \multirow{2}{*}{1458} & Age adj. & $1 \cdot 23(1 \cdot 16-1 \cdot 29)$ \\
\hline & & & & Multi adj. & $1 \cdot 19(1 \cdot 13-1 \cdot 26)$ \\
\hline & \multirow{2}{*}{ Undiagnosed DM } & \multirow{2}{*}{78134} & \multirow{2}{*}{881} & Age adj. & $1 \cdot 31(1 \cdot 22-1 \cdot 40)$ \\
\hline & & & & Multi adj. & $1 \cdot 23(1 \cdot 15-1 \cdot 32)$ \\
\hline & \multirow{2}{*}{ Diagnosed DM } & \multirow{2}{*}{77314} & \multirow{2}{*}{715} & Age adj. & $1 \cdot 37(1 \cdot 28-1 \cdot 48)$ \\
\hline & & & & Multi adj. & $1 \cdot 30(1 \cdot 21-1 \cdot 41)$ \\
\hline \multirow{10}{*}{ } & Glucose level & PYR & Events & Adjustment & HR $(95 \%$ CI) \\
\hline & \multirow{2}{*}{ Low } & \multirow{2}{*}{148663} & \multirow{2}{*}{397} & Age adj. & $0 \cdot 95(0 \cdot 86-1 \cdot 05)$ \\
\hline & & & & Multi adj. & $0.99(0.90-1.09)$ \\
\hline & Normal & 5166911 & 21145 & Ref. & $1 \cdot 00$ \\
\hline & \multirow{2}{*}{ IFG } & \multirow{2}{*}{162318} & \multirow{2}{*}{1612} & Age adj. & $1 \cdot 57(1 \cdot 49-1 \cdot 65)$ \\
\hline & & & & Multi adj. & $1 \cdot 40(1 \cdot 33-1 \cdot 48)$ \\
\hline & \multirow{2}{*}{ Undiagnosed DM } & \multirow{2}{*}{76669} & \multirow{2}{*}{1406} & Age adj. & $2 \cdot 52(2 \cdot 38-2 \cdot 66)$ \\
\hline & & & & Multi adj. & $2 \cdot 11(1 \cdot 99-2 \cdot 23)$ \\
\hline & \multirow{2}{*}{ Diagnosed DM } & \multirow{2}{*}{75983} & \multirow{2}{*}{1044} & Age adj. & $2 \cdot 54(2 \cdot 39-2 \cdot 70)$ \\
\hline & & & & Multi adj. & $2 \cdot 22(2 \cdot 08-2 \cdot 36)$ \\
\hline \multirow{10}{*}{ 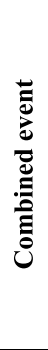 } & Glucose level & PYR & Events & Adjustment & HR $(95 \%$ CI) \\
\hline & \multirow{2}{*}{ Low } & \multirow{2}{*}{146578} & \multirow{2}{*}{717} & Age adj. & $0.98(0.91-1.06)$ \\
\hline & & & & Multi adj. & $1 \cdot 00(0 \cdot 93-1 \cdot 08)$ \\
\hline & Normal & 5070759 & 35929 & Ref. & $1 \cdot 00$ \\
\hline & \multirow{2}{*}{ IFG } & 157797 & 2336 & Age adj. & $1 \cdot 37(1 \cdot 32-1 \cdot 43)$ \\
\hline & & & 2050 & Multi adj. & $1 \cdot 28(1 \cdot 23-1 \cdot 34)$ \\
\hline & Undiagnosed DM & 74535 & 1745 & Age adj. & $1 \cdot 88(1 \cdot 80-1 \cdot 98)$ \\
\hline & & & 1745 & Multi adj. & $1 \cdot 67(1 \cdot 59-1 \cdot 76)$ \\
\hline & Diagnosed DM & 74069 & 1356 & Age adj. & $1 \cdot 92(1 \cdot 82-2 \cdot 03)$ \\
\hline & & & & Multi adj. & $1 \cdot 75(1 \cdot 65-1 \cdot 85)$ \\
\hline
\end{tabular}

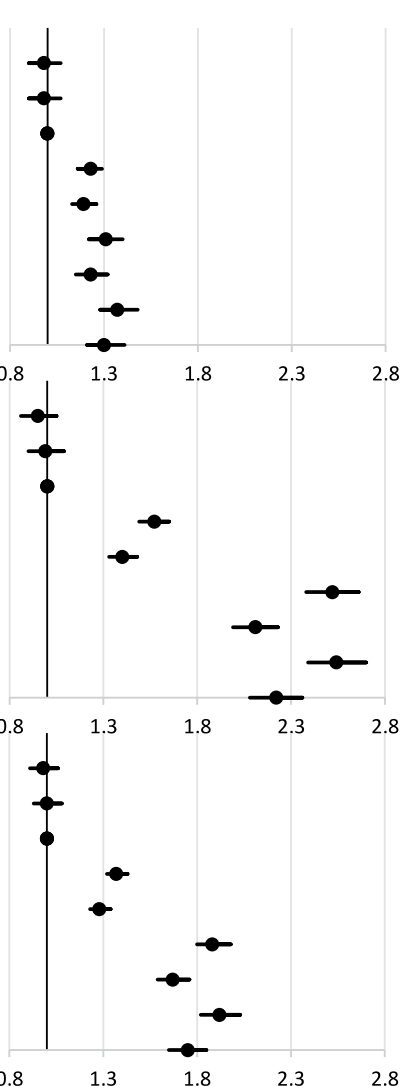

Fig. 2 Total events, and hazard ratios (HR) for atrial fibrillation, heart failure and combined event by glucose group. Hazard ratios are presented as adjusted for attained age as timescale and sex (Age adj.) and further adjusted for total cholesterol, total triglyceride levels and socioeconomic index (Multi adj.). PYR Person-years under risk, IFG Impaired fasting glucose, DM Diabetes mellitus

heart failure, even at levels below the diagnostic diabetes threshold. Second, the association between glucose and heart failure is stronger than the association to atrial fibrillation. Third, the association between glucose and atrial fibrillation could at least in part be due to or mediated by increased BMI, i.e. overweight and obesity, main risk factors of impaired fasting glucose and type 2 diabetes.

The main strength of this study is the large size of this population-based cohort without previous cardiovascular disease. The cohort was followed for a mean of nearly two decades, with high quality national registries ensuring essentially no loss of follow-up. Furthermore, all blood samples were analyzed on fresh blood in one single laboratory using consistently implemented and well documented methods, ensuring high quality and comparable measurements.

However, information on BMI was limited to a subpopulation and information on waist circumference was lacking, thus the sub-analyses adjusting for BMI must be interpreted with caution. Information on hypertension, current medication, blood pressure and other life-style factors was limited and could not be included. As these are all potential confounding factors, and hypertension an established risk factor for both atrial fibrillation and heart failure, this is an important limitation. Levels of HbA1c was only available for a small proportion of the cohort, as this was not routinely analyzed at the time of inclusion. Information on type of diabetes was not available. The majority of individuals with diabetes in Sweden at time of inclusion had type 2 diabetes, but since the risk for cardiovascular disease is higher in type 1 diabetes this could have caused a minor overestimation of risk. For identification of subjects with diabetes two national registries were used to achieve a high level of completeness. However, it is possible that some subjects with diabetes lacked a registered diagnosis. Since we did not have information regarding medication, it is possible that some subjects with well controlled diabetes and lack of registered diagnoses were misclassified as not having diabetes, which may have slightly diluted actual hazard ratios. Furthermore, information on type of heart failure was not available, but all cases of heart failure required either hospitalization or specialized outpatient care. As 
Table 2 Sensitivity analysis in a subgroup of 55390 subjects with BMI available, stratified by fasting glucose levels

\begin{tabular}{|c|c|c|c|c|}
\hline \multirow[t]{2}{*}{ Glucose level } & \multicolumn{2}{|c|}{$\begin{array}{l}\text { Atrial fibrillation } \\
4167 \text { events }\end{array}$} & \multicolumn{2}{|c|}{$\begin{array}{l}\text { Heart failure } \\
3138 \text { events }\end{array}$} \\
\hline & Events & $\mathrm{HR}[95 \% \mathrm{Cl}]$ & Events & $\mathrm{HR}[95 \% \mathrm{CI}]$ \\
\hline $\begin{array}{l}\text { Low } \\
n=1622(2.9 \%)\end{array}$ & 75 & $\begin{array}{l}0.88 \\
{[0.70-1.10]}\end{array}$ & 51 & $\begin{array}{l}0.88 \\
{[0.67-1.16]}\end{array}$ \\
\hline $\begin{array}{l}\text { Normal } \\
n=50,793(91.7 \%)\end{array}$ & 3713 & 1.00 & 2628 & 1.00 \\
\hline $\begin{array}{l}\text { IFG } \\
n=1537(2.8 \%)\end{array}$ & 187 & $\begin{array}{l}1.12 \\
{[0.96-1.30]}\end{array}$ & 175 & $\begin{array}{l}1.34 \\
{[1.15-1.57]}\end{array}$ \\
\hline $\begin{array}{l}\text { Undiagnosed DM } \\
\mathrm{n}=705(1.3 \%)\end{array}$ & 95 & $\begin{array}{l}1.25 \\
{[1.02-1.54]}\end{array}$ & 158 & $\begin{array}{l}2.82 \\
{[2.39-3.32]}\end{array}$ \\
\hline $\begin{array}{l}\text { Diagnosed DM } \\
\mathrm{n}=733(1.3 \%)\end{array}$ & 97 & $\begin{array}{l}1.36 \\
{[1.11-1.67]}\end{array}$ & 126 & $\begin{array}{l}2.54 \\
{[2.11-3.04]}\end{array}$ \\
\hline \multicolumn{5}{|c|}{ After further adjustment for BMI } \\
\hline $\begin{array}{l}\text { Low } \\
n=1622(2.9 \%)\end{array}$ & 75 & $\begin{array}{l}0.93 \\
{[0.74-1.17]}\end{array}$ & 51 & $\begin{array}{l}0.93 \\
{[0.70-1.22]}\end{array}$ \\
\hline $\begin{array}{l}\text { Normal } \\
n=50,793(91.7 \%)\end{array}$ & 3713 & 1.00 & 2628 & 1.00 \\
\hline $\begin{array}{l}\text { IFG } \\
n=1537(2.8 \%)\end{array}$ & 187 & $\begin{array}{l}1.03 \\
{[0.89-1.19]}\end{array}$ & 175 & $\begin{array}{l}1.25 \\
{[1.07-1.46]}\end{array}$ \\
\hline $\begin{array}{l}\text { Undiagnosed DM } \\
n=705(1.3 \%)\end{array}$ & 95 & $\begin{array}{l}1.11 \\
{[0.90-1.36]}\end{array}$ & 158 & $\begin{array}{l}2.51 \\
{[2.12-2.96]}\end{array}$ \\
\hline $\begin{array}{l}\text { Diagnosed DM } \\
n=733(1.3 \%)\end{array}$ & 97 & $\begin{array}{l}1.25 \\
{[1.02-1.53]}\end{array}$ & 126 & $\begin{array}{l}2.36 \\
{[1.96-2.83]}\end{array}$ \\
\hline
\end{tabular}

Hazard ratios and 95\% confidence limits for the events adjusted for

attained age as timescale, sex, total cholesterol, total triglyceride levels and socioeconomic index shown on top with further adjustment for BMI below IFG Impaired fasting glucose, DM Diabetes mellitus

the majority (92\%) of subjects were born in a Nordic country it is likely that the ethnicity was mainly Caucasian, limiting the generalizability of this study.
It is well known from several epidemiological cohort studies that glucose levels even below the diagnostic diabetes threshold are associated with coronary heart disease, cardiovascular mortality and total mortality [21]. The present study shows that there also is a graded association between glucose and atrial fibrillation and heart failure, starting already at prediabetes levels. This is also supported in a recent meta-analysis on cohort studies in diabetes and prediabetes [16]. However, we believe that our finding of a continuous association of glucose levels and future risk for atrial fibrillation in persons free from previous cardiovascular disease is unique with regards to the large cohort-size and extensive follow-up time and in the same cohort showing associated risks for heart failure. Importantly, atrial fibrillation was present not only in conjunction with or following heart failure, but in the majority of subjects appeared before or in the absence of heart failure. At ages from 65 years and above there was an equal distribution between heart failure and atrial fibrillation as the first occurring event, but in younger subjects atrial fibrillation more frequently appeared as the first of these two outcomes.

Subjects with elevated glucose had higher total cholesterol, triglycerides and apoB-apoA-1 ratios. Information on BMI was available in a subpopulation, among which BMI was higher in all groups with elevated glucose levels. As the association between elevated glucose and in particular atrial fibrillation was attenuated when further adjustment for BMI was made, this could indicate that part of the associated risk might be explained or mediated by obesity and/or presence of the metabolic syndrome. However, as information on BMI was only available in $19 \%$ of the cohort and was not consistently
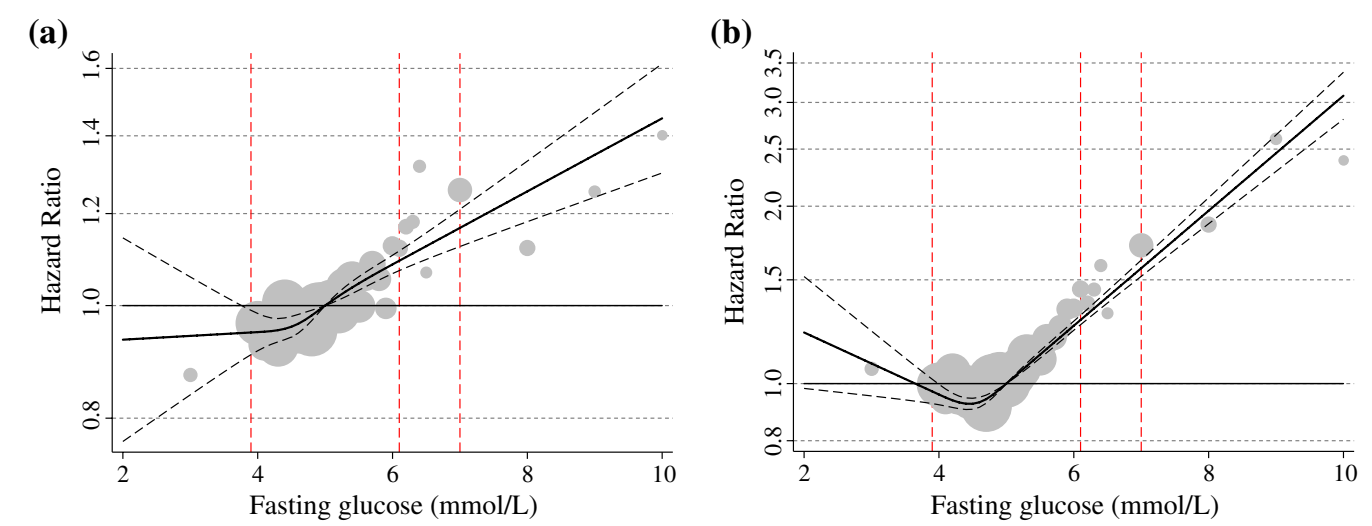

Fig. 3 Hazard ratio and 95\% confidence limits for $\mathbf{A}$ atrial fibrillation (left) and $\mathbf{B}$ heart failure (right) by fasting glucose level presented as splines. Subjects with a fasting glucose above or below 10 standard deviations not presented. Hazard ratios are adjusted for attained age as timescale, sex, total cholesterol, total triglyceride levels and socioeconomic index. The dotted lines indicate the 95\% confidence interval. The grey spheres reflect the relative size of the number of subjects with respective level of fasting glucose and the red vertical lines show the cut-off value for low glucose (3.9 $\mathrm{mmol} / \mathrm{L})$, and the lower and higher limit for impaired fasting glucose $(6.1 \mathrm{mmol} / \mathrm{L}$ and $6.9 \mathrm{mmol} / \mathrm{L})$ respectively 

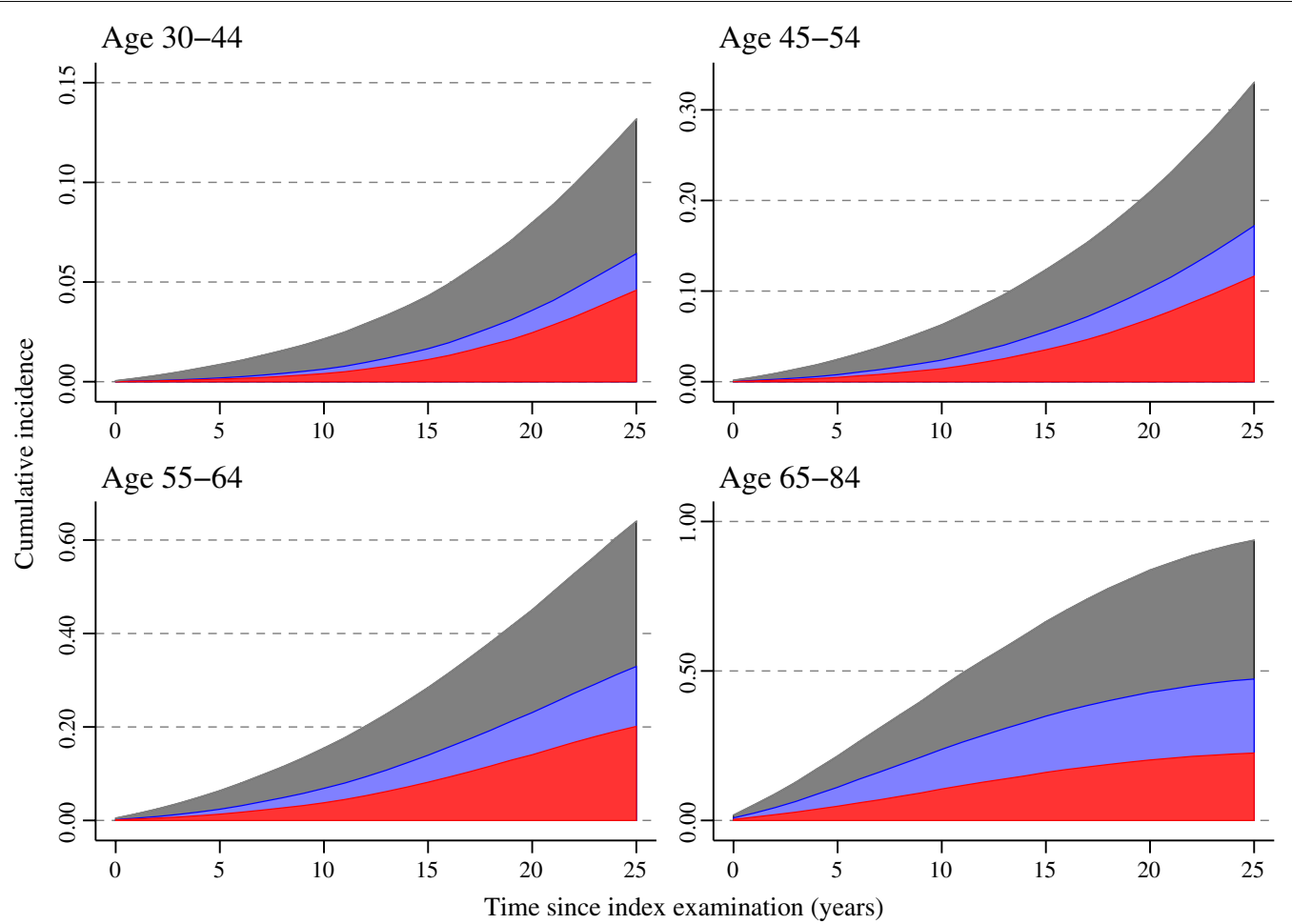

Time since index examination (years)

Atrial fibrillation $\square$ Heart failure

Death, other

Fig. 4 Cumulative incidence of events. Cumulative incidence (shown as surface area of each curve) for men and women of first event of atrial fibrillation (red), heart failure (blue) or death due to other causes (black) during 25 years of follow-up in different age groups. Observe the different scales on the $y$-axis between the age groups

recorded at the same date as the glucose level, these results must be interpreted with caution.

The association of diabetes, the metabolic syndrome and obesity to atrial fibrillation has recently gained increased interest $[7,22]$ with latest ESC guidelines for the management of atrial fibrillation emphasizing lifestyle interventions, including weight control [23]. There are several possible mechanistic explanations for such a relationship. These include atrial remodeling with increased fibrosis [24] and enlarged atrial volume associated with obesity [25]. Factors associated with the metabolic syndrome, such as hypertension, chronic inflammation, increased circulating lipids/free fatty acids, hyperinsulinemia and unhealthy adipose tissue distribution could be contributing pathophysiological mechanisms apart from direct glucose effects [7, 26]. Further, increased pericardial fat accumulation might disturb atrial function [25, 27]. Development of diastolic dysfunction and heart failure with preserved ejection fraction, elevated filling pressures and volume overload of the left atrium could be other potential drivers for the association between dysglycaemic conditions with and without co-existing obesity and atrial fibrillation [22, 28].
The association between elevated glucose levels and heart failure seemed to be stronger than the association to atrial fibrillation, regardless of glucose group. A speculative explanation could be that diabetes affects a majority of important functions and structures involved in the development of heart failure more than atrial fibrillation, as seen in diabetic cardiomyopathy (including myocardial dysmetabolism, coronary heart disease, myocardial fibrosis and hypertrophy) [28].

Of special interest in relation to the association of glucose and atrial fibrillation, is the recently highlighted possibility to prevent development of atrial fibrillation by modifying risk factors associated with dysglycaemia, the metabolic syndrome and a sedentary lifestyle. The ARREST-AF study showed that multifactorial life-style changes in patients with atrial fibrillation resulted in reduced recurrence of atrial fibrillation [29]. In the LEGACY study, weight loss reduced the burden of recurrent atrial fibrillation with contemporary improvement in echocardiographic abnormalities [30]. However, few, if any, have studied primary prevention of atrial fibrillation by modifying dysglycaemia and/or the metabolic syndrome. Interestingly, treatment with SGLT-2 inhibitors 
among patients with type 2 diabetes seems to decrease development of atrial fibrillation in the DECLARE-TIMI 58 study [31]. A recent retrospective study of patients newly diagnosed with type 2 diabetes and subsequently prescribed SGLT-2 inhibitors showed that the risk for incident atrial fibrillation was significantly lowered, but only among those who achieved a weight loss of of more than $5 \%$ [32], implicating the potential to reduce the future prevalence of atrial fibrillation through preventive treatment.

Considering that dysglycaemia is a global epidemic, and that the incidence of atrial fibrillation is increasing worldwide [33], our results emphasize the need for preventive action. There is a need for future studies exploring whether improved glycemic control in individuals with prediabetes affect the risk of future cardiovascular disease.

\section{Conclusions}

Elevated fasting glucose and unrecognized diabetes are risk factors for future development of both atrial fibrillation and heart failure, strengthening that early detection of dysglycaemia is an important risk factor to consider in cardiovascular prevention.

\section{Supplementary Information}

The online version contains supplementary material available at https://doi. org/10.1186/s12933-021-01422-3.

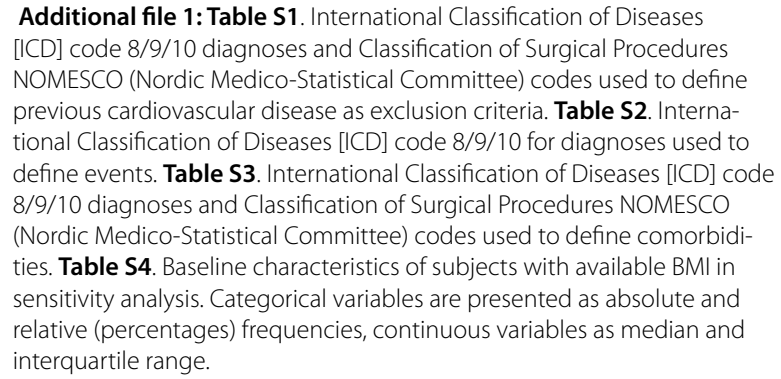

\section{Acknowledgements}

Not applicable.

\section{Authors' contributions}

All authors, VL, NH, LF, GW, PL, MT, AN developed the study design. AN and $\mathrm{NH}$ initiated the first study hypothesis. $V L$ and $A N$ finalized the manuscript after amendments by all authors. NH and GW initiated the AMORIS cohort. MT managed the database and performed the statistical analyses. All authors $V L$, $\mathrm{NH}, \mathrm{LF}, \mathrm{GW}, \mathrm{PL}, \mathrm{MT}$, AN made substantial contributions to this paper and took part in the interpretation of the results. All authors read and approved the final manuscript.

\section{Funding}

Open access funding provided by Karolinska Institute. This work was supported by The Swedish Heart and Lung Foundation, Stockholm, Sweden, grant numbers 20180524, 20170367, to AN; the Hjärtat foundation at Danderyds hospital, Stockholm, Sweden; and the Gunnar \& Ingmar Jungner Foundation of Laboratory Medicine, Stockholm, Sweden. The study funders were not involved in the design of the study; the collection, analysis, and interpretation of data; writing the report; and did not impose any restrictions regarding the publication of the report.

\section{Availability of data and materials}

Anonymized personal data were obtained from national Swedish Registry holders after ethical approval and secrecy assessment. According to Swedish laws and regulations, personal sensitive data can only be made available for researchers who fulfil legal requirements for access to personal sensitive data. Contact Professor Niklas Hammar (niklas.hammar@ki.se) for questions about data access.

\section{Declarations}

\section{Ethics approval and consent to participate}

This study complies with the Declaration of Helsinki and was approved by the Regional Ethical Review Board in Stockholm (Reg.No. 2010/1047-31/1). No written consent was required.

\section{Consent for publication \\ Not applicable.}

\section{Competing interests}

The authors declare that they have no conflicts of interest concerning this article. $V L$ has nothing to disclose. $\mathrm{NH}$ has nothing to disclose. PL reports personal fees from Boehringer Ingelheim, personal fees from Sanofi, personal fees from Novo Nordisk, outside the submitted work. LF reports personal fees from Bayer, personal fees from Boehringer Ingelheim, personal fees from BMS, personal fees from Pfizer, personal fees from Sanofi, outside the submitted work. MT has nothing to disclose. GW has nothing to disclose. AN reports personal fees from Astra Zeneca, personal fees from MSD, personal fees from Boehringer Ingelheim, personal fees from Lilly, personal fees from Novo Nordisk, outside the submitted work.

\section{Author details}

${ }^{1}$ Division of Cardiovascular Medicine, Department of Clinical Sciences, Danderyd Hospital, Karolinska Institutet, 18288 Stockholm, Sweden. ${ }^{2}$ Department of Cardiology, Danderyd University Hospital, Stockholm, Sweden. ${ }^{3}$ Unit of Epidemiology, Institute of Environmental Medicine, Karolinska Institutet, Stockholm, Sweden. ${ }^{4}$ Cardiology Unit, Department of Medicine K2, Karolinska Institutet, Stockholm, Sweden. ${ }^{5}$ Capio S:T Görans Hospital, Stockholm, Sweden.

Received: 4 October 2021 Accepted: 15 November 2021

Published online: 24 November 2021

\section{References}

1. Rawshani A, Rawshani A, Franzén S, Sattar N, Eliasson B, Svensson AM, et al. Risk factors, mortality, and cardiovascular outcomes in patients with type 2 diabetes. N Engl J Med. 2018;379(7):633-44.

2. The Emerging Risk Factors Collaboration, Sarwar N, Gao P, Seshasai SR, Gobin R, Kaptoge S, et al. Diabetes mellitus, fasting blood glucose concentration, and risk of vascular disease: a collaborative meta-analysis of 102 prospective studies. Lancet. 2010; 375(9733):2215-22.

3. International Diabetes Federation. IDF Diabetes Atlas, 9th edn. 2019. https://diabetesatlas.org/atlas/ninth-edition/. Accessed 30 Apr 2020.

4. Cavender MA, Steg PG, Smith SC, Eagle K, Ohman EM, Goto S, et al. Impact of diabetes mellitus on hospitalization for heart failure, cardiovascular events, and death: outcomes at 4 years from the Reduction of Atherothrombosis for Continued Health (REACH) registry. Circulation. 2015;132(10):923-31.

5. Norhammar A, Bodegård J, Nyström T, Thuresson M, Eriksson JW, Nathanson D. Incidence, prevalence and mortality of type 2 diabetes requiring glucose-lowering treatment, and associated risks of cardiovascular complications: a nationwide study in Sweden, 2006-2013. Diabetologia. 2016;59(8):1692-701. 
6. Groenewegen A, Zwartkruis WW, Cekic B, de Boer RA, Rienstra M, Hoes $A W$, et al. Incidence of atrial fibrillation, ischaemic heart disease and heart failure in patients with diabetes. Cardiovasc Diabetol. 2021;20(1):123.

7. Wang A, Green JB, Halperin JL, Piccini JP. Atrial fibrillation and diabetes mellitus. J Am Coll Cardiol. 2019;74(8):1107-15.

8. Benjamin EJ, Levy D, Vaziri SM, D'Agostino RB, Belanger AJ, Wolf PA. Independent risk factors for atrial fibrillation in a population-based cohort. The Framingham Heart Study. JAMA. 1994;271(11):840-4.

9. Karayiannides S, Lundman P, Friberg L, Norhammar A. High overall cardiovascular risk and mortality in patients with atrial fibrillation and diabetes: a nationwide report. Diabetes Vasc Dis Res. 2018;15(1):31-8.

10. Donnellan E, Aagaard P, Kanj M, Jaber W, Elshazly M, Hoosien M, et al. Association between pre-ablation glycemic control and outcomes among patients with diabetes undergoing atrial fibrillation ablation. JACC Clin Electrophysiol. 2019;5(8):897-903.

11. Aune $D$, Schlesinger $S$, Neuenschwander M, Feng T, Janszky I, Norat $T$, et al. Diabetes mellitus, blood glucose and the risk of heart failure: a systematic review and meta-analysis of prospective studies. Nutr Metab Cardiovasc Dis. 2018;28(11):1081-91.

12. Wang J, Sarnola K, Ruotsalainen S, Moilanen L, Lepistö P, Laakso M, et al. The metabolic syndrome predicts incident congestive heart failure: a 20-year follow-up study of elderly finns. Atherosclerosis. 2010;210(1):237-42

13. Latini R, Staszewsky L, Sun JL, Bethel MA, Disertori M, Haffner SM, et al Incidence of atrial fibrillation in a population with impaired glucose tolerance: the contribution of glucose metabolism and other risk factors. A post hoc analysis of the Nateglinide and Valsartan in Impaired Glucose Tolerance Outcomes Research trial. Am Heart J. 2013;166(5):935-40.

14. Watanabe H, Tanabe N, Watanabe T, Darbar D, Roden DM, Sasaki S, et al. Metabolic syndrome and risk of development of atrial fibrillation: the Niigata preventive medicine study. Circulation. 2008;117(10):1255-60.

15. Huxley RR, Lopez FL, Folsom AR, Agarwal SK, Loehr LR, Soliman EZ, et al. Absolute and attributable risks of atrial fibrillation in relation to optimal and borderline risk factors. Circulation. 2011;123(14):1501-8.

16. Aune D, Feng T, Schlesinger S, Janszky I, Norat T, Riboli E. Diabetes mellitus, blood glucose and the risk of atrial fibrillation: a systematic review and meta-analysis of cohort studies. J Diabetes Complications. 2018;32(5):501-11.

17. Walldius $G$, Malmström $H$, Jungner I, de Faire $U$, Lambe $M$, Van HM, et al. Cohort profile: the AMORIS cohort. Int J Epidemiol. 2017;46(4):1103-1103i.

18. Malmström H, Walldius G, Carlsson S, Grill V, Jungner I, Gudbjörnsdottir S, et al. Elevations of metabolic risk factors 20 years or more before diagnosis of type 2 diabetes: experience from the AMORIS study. Diabetes Obes Metab. 2018;20(6):1419-26.

19. World Health Organization, International Diabetes Federation. Definition and diagnosis of diabetes mellitus and intermediate hyperglycaemia: Report of a WHO/IDF consultation 2006. https://www.who.int/diabetes/ publications/Definition\%20and\%20diagnosis\%20of\%20diabetes_new. pdf. Accessed 30 Apr 2020.

20. Levey AS, Stevens LA, Schmid CH, Zhang Y, Castro AF, Feldman HI, et al. A new equation to estimate glomerular filtration rate. Ann Intern Med. 2009;150(9):604-12.
21. Coutinho M, Gerstein HC, Wang Y, Yusuf S. The relationship between glucose and incident cardiovascular events: A metaregression analysis of published data from 20 studies of 95,783 individuals followed for 12.4 years. Diabetes Care. 1999;22(2):233-40.

22. Packer M. Do most patients with obesity or type 2 diabetes, and atrial fibrillation, also have undiagnosed heart failure? A critical conceptual framework for understanding mechanisms and improving diagnosis and treatment. Eur J Heart Fail. 2020;22(2):214-27.

23. Hindricks G, Potpara T, Dagres N, Bax JJ, Boriani G, Dan GA, et al. 2020 ESC Guidelines for the diagnosis and management of atrial fibrillation developed in collaboration with the European Association for Cardio-Thoracic Surgery (EACTS). Eur Heart J. 2021;42(5):373-498.

24. Russo I, Frangogiannis NG. Diabetes-associated cardiac fibrosis: cellular effectors, molecular mechanisms and therapeutic opportunities. J Mol Cell Cardiol. 2016;90:84-93.

25. Singh M, Sethi A, Mishra AK, Subrayappa NK, Stapleton DD, Pellikka PA. Echocardiographic imaging challenges in obesity: guideline recommendations and limitations of adjusting to body size. J Am Heart Assoc. 2020;9(2):e014609.

26. Tadic M, Cuspidi C. Type 2 diabetes mellitus and atrial fibrillation: from mechanisms to clinical practice. Arch Cardiovasc Dis. 2015;108(4):269-76.

27. Greif M, Von ZF, Wakili R, Tittus J, Becker C, Helbig S, et al. Increased pericardial adipose tissue is correlated with atrial fibrillation and left atrial dilatation. Clin Res Cardiol. 2013;102(8):555-62.

28. Seferović PM, Paulus WJ. Clinical diabetic cardiomyopathy: a twofaced disease with restrictive and dilated phenotypes. Eur Heart J. 2015;36(27):1718-27.

29. Pathak RK, Middeldorp ME, Lau DH, Mehta AB, Mahajan R, Twomey $D$, et al. Aggressive risk factor reduction study for atrial fibrillation and implications for the outcome of ablation: the ARREST-AF cohort study. J Am Coll Cardiol. 2014;64(21):2222-31.

30. Pathak RK, Middeldorp ME, Meredith M, Mehta AB, Mahajan R, Wong CX, et al. Long-term effect of goal-directed weight management in an atrial fibrillation cohort. J Am Coll Cardiol. 2015;65(20):2159-69.

31. Zelniker TA, Bonaca MP, Furtado RHM, Mosenzon O, Kuder JF, Murphy SA, et al. Effect of dapagliflozin on atrial fibrillation in patients with type 2 diabetes mellitus: Insights from the DECLARE-TIMI 58 Trial. Circulation. 2020;141(15):1227-34.

32. Chan YH, Chen SW, Chao TF, Kao YW, Huang CY, Chu PH. The impact of weight loss related to risk of new-onset atrial fibrillation in patients with type 2 diabetes mellitus treated with sodium-glucose cotransporter 2 inhibitor. Cardiovasc Diabetol. 2021;20(1):93.

33. Hajhosseiny R, Matthews GK, Lip GYH. Metabolic syndrome, atrial fibrillation, and stroke: tackling an emerging epidemic. Heart Rhythm. 2015;12(11):2332-43.

\section{Publisher's Note}

Springer Nature remains neutral with regard to jurisdictional claims in published maps and institutional affiliations.

Ready to submit your research? Choose BMC and benefit from

- fast, convenient online submission

- thorough peer review by experienced researchers in your field

- rapid publication on acceptance

- support for research data, including large and complex data types

- gold Open Access which fosters wider collaboration and increased citations

- maximum visibility for your research: over $100 \mathrm{M}$ website views per year

At BMC, research is always in progress.

Learn more biomedcentral.com/submissions 